\section{$\underset{\substack{\text { hommes } \\ \text { \& migrations }}}{ }$}

\section{Hommes \& migrations}

Revue française de référence sur les dynamiques

migratoires

\section{$1283 \mid 2010$}

Cuisines et dépendances

\title{
Un métissage franco-turc des habitudes alimentaires
}

Au menu des adolescents originaires de Turquie

\section{Hatice Soyturk}

\section{OpenEdition}

Journals

Édition électronique

URL : http://journals.openedition.org/hommesmigrations/989

DOI : 10.4000/hommesmigrations.989

ISSN : 2262-3353

Éditeur

Musée national de l'histoire de l'immigration

Édition imprimée

Date de publication : 1 janvier 2010

Pagination : 52-61

ISBN : 978-2-919040-04-9

ISSN : 1142-852X

Référence électronique

Hatice Soyturk, "Un métissage franco-turc des habitudes alimentaires », Hommes \& migrations [En

ligne], 1283 | 2010, mis en ligne le 29 mai 2013, consulté le 01 mai 2019. URL : http://

journals.openedition.org/hommesmigrations/989; DOI : 10.4000/hommesmigrations.989 


\section{Au menu des adolescents originaires de Turquie Un métissage franco-turc des habitudes alimentaires}

Par Hatice Soyturk, socio-anthropologue, assistante-chercheur au laboratoire Cultures et sociétés en Europe (CNRS-université de Strasbourg)

Pour les adolescents d'origine turque en France, l'alimentation et la préparation des plats constituent un marqueur identitaire fort. La cuisine turque est également, pour les jeunes, l'objet d'une constante expérimentation. La sélection des pratiques, des plats et de leur mélange ne cesse d'évoluer. Des kofte et autres dolma aux hamburgers se joue le portrait d'une assiette métissée qui négocie sa place dans la société française. 
Limmigration turque en France semble multiple et plurielle, tant au niveau culturel que religieux : Alévis, Sunnites, Kurdes, Turcs... Ils emportent tous dans leurs bagages des aliments, des ustensiles de cuisine, des pratiques alimentaires et des savoir-faire culinaires qu'ils vont chercher à transmettre à leurs enfants et petits-enfants, et à transplanter dans la société française. Les modalités de cette transplantation peuvent différer en fonction des populations concernées, des contextes et des trajectoires migratoires. C'est ce que nous avons vu en esquissant l'espace de consommations alimentaires des adolescents originaires de Turquie.

En Alsace, une étude socio-anthropologique dans le cadre du projet AlimAdos ${ }^{(1)}$, sur les comportements alimentaires des adolescents dont les parents sont originaires de Turquie, nous a plongé dans un univers de diversité. N. Elias ${ }^{(2)}$ montre qu'il est impossible d'isoler le comportement à table du contexte social ${ }^{(3)}$. Par son matériau, l'étude montre que l'alimentation est à mettre en relation avec des phénomènes politiques, économiques, sociaux et culturels. En effet, l'évolution du domaine alimentaire en France relève aussi de la commercialisation de produits dits "exotiques", ainsi que des nouvelles formes de distribution. Les stratégies mises en place pour le développement de commerces turcs en Alsace et des services comme la restauration turque ont permis aux migrants de Turquie et à leurs héritiers de conserver leurs pratiques alimentaires et culinaires ou de les adapter aux conditions nouvelles. Les produits turcs importés au fil des années en Europe se retrouvent aujourd'hui comme marqueurs d'un certain exotisme ${ }^{(4)}$.

\section{La diaspora turque dans l'assiette}

Nos observations nous ont permis de voir se dessiner un espace social à la croisée des cultures. En effet, les familles originaires de Turquie font découvrir leur gastronomie à la population autochtone en suscitant notamment des rencontres par le biais des activités associatives. L'étude montre que l'ensemble des pratiques alimentaires et culinaires constitue une part du patrimoine des migrants originaires de Turquie et de leurs héritiers.

Les enjeux de leur alimentation et de leurs pratiques culinaires dépassent la seule dimension pratique pour recouvrir des valeurs identitaires. Ils traduisent les relations entre migrants, grands-parents, parents et adolescents, les liens avec le pays d'origine et reflètent les trajectoires migratoires de chacun. Ils accompagnent donc les processus identitaires dans toute leur ampleur. Toutefois, les pratiques alimentaires et culinaires de la population turque subissent un processus d'arrangement ${ }^{(5)}$, par les contacts incessants avec les pratiques et objets culinaires de la société française. 
Les adolescents dont les parents sont originaires de Turquie, à l'instar de leurs pairs, se construisent en tant qu'individus par une recomposition des références identitaires dans leurs pratiques alimentaires. Tout autant, voire plus que leurs parents ou grands-parents, ils tiennent à la "tradition culinaire" (il s'agit des plats turcs, des plats cuisinés comme en Turquie ou alors des plats de leurs grands-mères), sans que cela soit en concurrence avec la consommation d'aliments propres à leur classe d'âge (12-19 ans).

Ces recompositions culturelles, qui se jouent entre mémoire familiale et immédiateté de l'appartenance au groupe des adolescents, peuvent être vues comment autant d'analyseurs des différentes façons dont ils conçoivent leur turcité ou kurditét(6) et, par là, leur rapport à la société française d'aujourd'hui.

Ces différentes façons d'imaginer leur appartenance à la communauté turque ou kurde se produiraient dans un rapport complexe de proximité et de distance à l'égard du pays d'origine et de la société dans laquelle ils vivent. Cette dialectique se polarise entre ici et là-bas, et cette polarisation est pertinente pour décrire les visions du monde des adolescents puisqu'elles transparaissent dans les pratiques alimentaires et culinaires. Les comportements alimentaires des jeunes originaires de Turquie, les mélanges dans les assiettes, l'émergence de nouvelles sauces, la disposition des tables ("à la turque" ou "à la française") apparaissent ainsi comme autant d'indicateurs d'une rencontre des cultures, et partant, d'un métissage plus large, dépassant le seul cadre des assiettes.

\section{Richesse et diversité de la cuisine turque en France}

Kevin est un adolescent de 17 ans, originaire de l'est de l'Anatolie, appartenant au groupe kurde. Ses réflexions sur ses comportements et habitudes alimentaires, mais aussi sur le lien qu'il fait entre son alimentation et son appartenance au groupe turc ou kurde, le singularisent au sein d'un groupe d' adolescents. Il a essayé de voir, de comprendre, de réfléchir à ce qu'il mange et de démontrer comment, par le biais de l'alimentation qu'il qualifie tantôt de "turque", tantôt de "kurde", se vit une part de son identité et de son appartenance au groupe kurde ${ }^{(7)}$. "Mon alimentation correspond à la nourriture du village, les plats traditionnels. C'est riche. Mais quand je dis 'plats traditionnels', je ne parle pas que de la cuisine turque avec les soupes, les mézès, les kebab, les dolma ou les desserts. Je fais aussi allusion à mes origines. Par exemple, chez nous, on mange du ' ir'. C'est un plat kurde. C'est fait à base de beurre, de yaourt, d'ail et c'est cuit au four." 
Les propos de Kevin montrent bien la richesse ${ }^{(8)}$ de la cuisine turque, car la Turquie elle-même est multiethnique et vaste sur le plan géographique. Se définissant comme kurde au sein de cette diversité, il témoigne aussi d'une kurdité revendiquée. Selon lui, "la cuisine turque est constituée de plusieurs styles de cuisine : la cuisine des immigrés, par exemple, le style stambouliote ou encore les repas traditionnels du village. La cuisine turque est très riche et change en fonction des populations se trouvant en Turquie, mais elle change aussi grâce aux migrations." Cette distinction des aliments selon leur style montre la complexité du manger turc, la richesse et les changements en fonction des contextes et des lieux.

Kevin est conscient des changements culinaires en migration et perçoit ces changements dans ses pratiques. "Ma grand-mère cuisine ce qu'elle a vu depuis des années. C'est un héritage qu'elle a gardé, mais qu'elle transmet aussi. J'aime passer du

Face aux autres, tout en affirmant sa kurdité, cet adolescent utilise le hamburger et la marque Adidas comme preuve d'intégration à la société française. temps avec elle dans la cuisine. Elle reste très fidèle aux repas traditionnels : elle ne met pas de ketchup, par exemple, ou de mayonnaise dans un plat turc. Elle sait que je n'aime pas faire de mélanges. Mais elle n'aime pas varier comme moi. Elle ne mange pas européen, ni hamburgers ni pizzas. Elle fait des pizzas turques, des Lahmacun. C'est différent. On n'a pas la même culture. Moi, même si je suis d'origine kurde, je ne mange pas que des aliments kurdes ou turcs."

\section{Traditions culinaires et regards des autres : le choix du va-et-vient}

Kevin utilise souvent le mot "héritage". Selon lui, cet héritage s'inscrit notamment dans les "techniques de cuisson" que sa grand-mère lui a apprises. Ce sont non seulement les aliments, mais aussi des manières de faire et la tradition qui changent. La transmission porte principalement là-dessus. Derrière cette identification au groupe turc ou kurde, nous observons aussi de multiples pratiques liées à l'expérience migratoire. Kevin se distingue de ses parents et de ses grands-parents.

"Nous, les garçons, on aime manger au MacDo. Je ne sais pas pourquoi, mais on préfêre les hamburgers. C'est pas mieux que les döner, ça c'est sûr, mais ça change. Et c'est mieux vu par les autres. Quand on dit qu'on a mangé un döner, on nous regarde avec de grands yeux, comme si c'était un signe qui montre que l'on n'est pas intégré. Moi jassume mes 
origines. Je suis Kurde, fier de l'être, mais, en même temps, jaime varier ce que je mange." Le MacDo, défini comme "international", est vu comme un moyen permettant de se distinguer des autres dans un conflit avec le döner, un aliment qui a tendance à être typique de la cuisine turque. Face aux autres, tout en affirmant sa kurdité, cet adolescent utilise le hamburger et la marque Adidas comme preuve d'intégration à la société française.

Il distingue cuisine européenne et héritage culinaire turc. Cette distinction met en évidence la pluralité des lieux de consommation, des aliments, des plats, des sauces, des mélanges, des manières de faire et d'agir, etc. "Il faut respecter les plats et les individus. On peut manger des aliments appartenant à différentes cultures pendant un même repas, mais il faut manger l'aliment tel qu'il est présenté et cuisiné, sans faire de rajout. Il ne faut pas mélanger les goûts. Sinon, on ne sait plus ce qu'on mange, ça devient n'importe quoi." L'exemple empirique de Kevin pointe non seulement la diversité en Turquie, mais aussi la diversité turque en diaspora. Il fait des va-et-vient entre l'"alimentation traditionnelle" et les plats communs qu'il qualifie souvent de "modernes".

En effet, en raison des phénomènes migratoires, les pratiques alimentaires de la population turque subissent un processus d'arrangement, et c'est notamment grâce aux facilités d'approvisionnement en Alsace que les Turcs préservent une tradition alimentaire néanmoins continuellement bousculée, ou réarrangée, par les incessants contacts avec les pratiques et les objets culinaires de la société d'accueil ${ }^{(9)}$. Le terrain turc et les analyses montrent que les plats turcs condensent plusieurs modalités techniques, mais aussi plusieurs âges correspondant aux strates de l'expérience diasporique. L'origine sociale et culturelle des parents, arrivés dans les années soixante-dix pour des raisons économiques, dans les années quatre-vingt pour des raisons politiques et dans les années quatre-vingt-dix pour des raisons familiales, influencent les comportements des adolescents et requièrent une distinction intergénérationnelle entre les grands-parents, les parents et les adolescents. Elle s'opère selon la préparation des plats, la combinaison des ingrédients et les manières de table.

\section{La mémoire de la table : ritualisation, codes et normes}

Les multiples repas partagés avec les adolescents et leurs familles montrent que la table est un lieu important où l'on observe le croisement des goûts ${ }^{(10)}$, des arômes, des paroles, des inquiétudes, des conflits, des gênes mais aussi des joies, de la convivialité et du partage. Les adolescents sont généralement tenus de prendre leur repas avec la 
famille, puisque l'éducation passe souvent par ce moment. Le repas en famille est inévitable, même si nous observons une individualisation des pratiques et des modes de consommation ${ }^{(11)}$. Lors des entretiens, les adolescents énumèrent souvent des règles concernant les repas en famille. Ils évoquent plusieurs points : l'horaire, l'ordonnancement des places accordées aux personnes, la répartition des rôles et des tâches, mais aussi l'apprentissage des manières de table et le respect. La table est aussi perçue comme un lieu de négociation des façons de faire et de se conduire. Pour la majorité des adolescents, "passer à table" signifie "prendre place". Les adolescents attendent souvent le père de famille pour passer à table. Sa parole et son silence ont un poids décisif. Dans certaines familles, la parole est réservée aux adultes. L'adolescent ne répond que lorsqu'une question lui est posée, mais, dans la majorité des familles turques observées, c'est à table que l'on se parle, que l'on échange. La parole à table met au courant des faits et des situations de chacun, mais des stratégies de détournement, comme la télé, sont aussi mises en place par les adolescents pour éviter certaines conversations, car la table est contraignante. C'est là que se joue l'autorité parentale.

En ce qui concerne les manières de table, il existe encore quelques familles qui ne disposent pas de salle à manger et qui continuent à prendre le repas comme le font les grands-parents restés au village. Autrement dit, elles possèdent une table basse et ronde qui semble être bien adaptée au repas turc, car elle permet à tous d'être à une distance égale du ou des plats placés au milieu. Attablés à ras du sol, les convives ne disposent pas toujours d'une assiette individuelle, mais doivent se servir une multitude de fois des aliments posés dans des plats communs. En résumé, la table reste le principal lieu de socialisation et de confrontation des adolescents de culture turque.

\section{L'alimentation familiale et les pratiques culinaires comme objets d'une transmission}

Lorsque nous abordons la question de la transmission familiale ${ }^{(12)}$, les adolescents font état de l'existence de dispositions culinaires familiales héritées des grandsparents, plus disponibles que les parents lorsqu'ils se trouvent en France. Les personnes âgées sont perçues comme les dépositaires d'une mémoire culinaire. La transmission familiale passe par une réappropriation des savoirs et des pratiques que les anciens ont appris au village d'origine. Les adolescents évoquent la question du "pur" et de l'“impur" lorsqu'ils parlent de la viande "helâl ou pas helâl". Ils 
s'attardent également sur des questions liées à la consommation d'alcool et sur les interdits religieux tels que l'interdiction de manger du porc. Ils mentionnent des gestuelles en évoquant des rituels ${ }^{(13)}$.

Aynur, une adolescente de 15 ans (Strasbourg) eplique: "Les pratiques [en lien avec la tradition] essayent de survivre. On essaye de nous transmettre des choses [en parlant des grands-parents]. Et puis, maintenant, ce n'est pas comme avant. Au village, nos grands-parents utilisaient du bois ou de la paille pour cuisiner. Ici, on fait tout au four." Gülay, une adolescente de 19 ans (Colmar), pense, quant à elle, qu'il y a bien une différence entre son mode de vie alimentaire et celui de ses grands-parents restés en Turquie. "Nous, on vit en ville. Eux, ils sont encore dans des zones rurales. Ce sont des campagnards. Ils mangent ce qu'ils cultivent. C'est plus restreint. Ils n'ont pas les moyens de sortir, de manger en ville, au restaurant. Ils mangent tout le temps des plats traditionnels. Ils

\section{La transmission familiale passe par une réappropriation des savoirs et des pratiques que les anciens ont appris au village d'origine.} préparent tout eux-mêmes. Ils ne cherchent pas à faire du bricolage comme nous."

Aybüke, une adolescente de 18 ans (Colmar), trouve que ses habitudes alimentaires sont très influencées par ses grands-parents, et c'est la raison pour

laquelle cette famille mange peu de riz. "Nos grands-parents ne connaissaient pas le riz, c'était un plat de riches. Comme on vient d'une famille de paysans, des agriculteurs anatoliens, on ne sait cuisiner que des plats à base de farine, de viande, de légumes et de bulgur. Nous, quand on cuisine que pour nous, on ne prépare les Sarma, les biber dolmasi qu'avec du blé concassé, c'est rare que l'on ajoute du riz."

\section{Quand modernité rime avec facilité}

Les adolescents reçoivent une mémoire culinaire en continuant à utiliser des dénominations turques pour désigner certains aliments. D’une génération à l'autre, les plats se refont en même temps qu'ils se transforment.

Les adolescents préparent des aliments qu'ils qualifient de "faciles": salades, soupes en sachet, simple réchauffage d'un plat surgelé ou déjà prêt. Ils qualifient certains plats de "difficiles", parce qu'ils ne veulent ou ne savent pas les préparer. Il s'agit souvent pour eux "de plats plus sophistiqués, de plats traditionnels, qui demandent un réel investissement'. Les mets qu'ils n'arrivent pas à préparer ou qu'ils ne veulent pas cuisiner sont définis comme "réservés à la mère ou à la grand-mère". Betül, une adolescente de 16 ans (Strasbourg), explique : "Le matin, je prends le petit- 
déjeuner chez moi. À midi, je rentre déjeuner toute seule. Et c'est encore moi qui prépare le repas. Ce sont souvent des plats faciles à cuisiner, comme des surgelés par exemple."

Certains adolescents veulent négocier avec leurs parents en introduisant au sein du foyer des aliments connus de tous, qu'ils qualifient de "modernes ${ }^{(14)}$ ". Les adolescents font allusion aux frites, aux pizzas, aux pâtes, aux poulets rôtis, etc. Les mélanges sont des sauces ajoutées aux aliments de base et aux aliments dits "traditionnels". En effet, certains parents ont des comportements alimentaires que les adolescents qualifient de "modernes" et ne semblent pas prendre conscience des représentations que leurs enfants peuvent se faire de cette soi-disant modernité. Aybüke dit à ce propos : "Il nous arrive aussi de faire du riz et de cuisiner des plats avec du riz, parce que c'est mieux vu par les gens qu'on connaît. Les invités préfêrent les plats turcs avec du riz. Un jour, ma mère avait préparé du dolma avec du blé, et on l'a prise pour une arriérée, comme si elle n'était pas moderne. Alors que ce n'est pas vrai. Ma mère sait s'adapter et nous, on préfêre des plats cuisinés à l'ancienne. C'est bien meilleur."

\section{Assiettes métisses : des traditions négociées et réinventées par les adolescents}

Les adolescents mettent l'accent sur la perpétuation des traditions culinaires tout en cherchant à gommer certaines spécificités. Nous ne remarquons pas de rejet fort des "nourritures traditionnelles", mais les remarques générales des adolescents montrent que l'alimentation turque commence à être un objet de dépréciation. Ils surfent entre aliments turcs et repas que nous pouvons qualifier d'“internationaux". Aux köfte, dolma, karnıyarık sont de plus en plus préférés les plats communs en France, comme les poulets rôtis, frites, pâtes, pizzas, hamburgers, etc. Ces plats ne se substituent pas complètement aux plats turcs mais viennent s'y ajouter. Les adolescents mêlent donc à la fois des aliments de leur culture d'origine avec ceux d'autres cultures. Pris dans le balancement des influences d'ici et des traditions culinaires de là-bas, ils composent leur "assiette turque". La cuisine turque commence à être mélangée avec celle de la société d'accueil.

Tayfun, un adolescent de 16 ans (Colmar), raconte : "Ma mère prépare une soupe au köfte, appelée 'köfte çorbası'. Comme salade, elle fait souvent la salade du berger, çoban salatas1, mais il peut lui arriver de faire une salade de pommes de terre, patates salatas1 très traditionnelle, mais avec de la mayonnaise. Au köy, au village, ils ne connaissent pas la mayonnaise. C'est ma mère qui en ajoute. Elle veut parfois se montrer 'moderne."' Les déclarations des adolescents révèlent une véritable conception turque de 
l'alimentation, défendue dans son modèle le plus "enraciné" et le plus traditionnel. Malgré cette référence à la tradition, comme nous l'avons vu, la cuisine des originaires de Turquie continue de changer au contact des sociétés d'accueil.

Le fait est que l'organisation de la cellule familiale des originaires de Turquie connaît d'importantes modifications et que les femmes sont de moins en moins disponibles pour la préparation des repas. En outre se développent des comportements de types grignotage, consommation de plats précuits, etc. Lorsque nous évoquons la question du métissage, les adolescents font d'ailleurs d'emblée un lien avec les mélanges qu'ils font dans leur assiette.

Gülay, par exemple, rapporte qu'il lui arrive de faire des échanges à table : "Mon petit frère aime faire des essais, il mélange le sucré et le salé, du coup ça donne un goût bizarre. Il me dit que c'est très bon et, avant de faire la même chose que lui, je goûte chez lui d'abord. Souvent, c'est bizarre. On dirait qu'il fait ça pour s'amuser, mais moi, je trouve que ce n'est pas marrant."

Tayfun (16 ans) a l'habitude de mélanger des plats turcs et non turcs contre l'avis de sa mère. "Je ne joue pas avec la nourriture, mais je la transforme. Ma mère me gronde souvent à table, parce que je prends trop de temps pour manger. Elle me dit: 'yemekle oynama!', 'ne joue pas avec la nourriture !'Mettre de la sauce harissa et de la mayonnaise sur les frites, et mélanger ça, pour elle, c'est jouer avec la nourriture." Ces mélanges, il les fait parce qu'il les aime. "C'est comme le Fanta et le Coca. Moi, je mélange ces deux boissons. C'est un goût différent, et c'est bon." Selon lui, il faut essayer pour comprendre. "Ma mère n'essaye pas, c'est pour ça qu'elle ne veut pas que je le fasse. Pour elle, il faut bien se tenir à table, ne pas discuter, surtout quand il y a mon père."

\section{Conclusion}

Pour conclure, nous pouvons dire que des formes d'identification se dessinent à partir des pratiques alimentaires et culinaires des adolescents. La nourriture et ses usages sociaux sont perçus comme des codes et comme des signes d'identité, c'est-àdire comme analyseurs d'un certain rapport au monde. L'aliment des adolescents est globalement perçu comme l'incarnation ou le reflet de leur culture d'origine, en l'occurrence celle de leurs parents et grands-parents. L'idée étant que le "manger turc" ne peut se comprendre détaché du contexte social de préparation des aliments. S'il y a un modèle du "manger turc", il est traversé par l'histoire et la mémoire de ceux qui le conçoivent et l'articulent à leurs pratiques. Lorsque ces pratiques changent ou se modifient, elles indiquent aussi pour les adolescents de nouvelles façons de percevoir leur identité. 


\section{Notes}

1. Le projet AlimAdos porte sur des jeunes de 12 à 19 ans issus d'horizons culturels et de milieux sociaux divers, en considérant les différences culturelles et les métissages à l'ceuvre dans la France multiculturelle d'aujourd'hui. L'étude, conduite avec deux laboratoires du CNRS, l'UMR 6578 de l'université de la Méditerranée à Marseille et l'UMR 7043 de l'université Marc Bloch à Strasbourg, compare six populations adolescentes : une population française (sans situation migratoire extranationale), maghrébine, turque, d'Afrique subsaharienne, d'Asie du Sud-Est et d'Europe orientale. L'objectif est de comprendre leurs habitudes alimentaires, tant dans leur diversité que dans ce qui caractérise une "culture adolescente" commune à tous. Cette recherche ambitieuse fait appel à des méthodes de diverses disciplines pour comprendre la relation des jeunes à leur corps et à leurs aliments dans les diverses situations de leur vie au quotidien, à l'école, en famille, et pendant leurs moments de loisirs, en week-ends et en vacances. Les chercheurs s'intéressent aussi aux proches de ces jeunes - parents, frères et sceurs, copains - et à l'environnement socio-éducatif responsables de restaurants scolaires, animateurs culturels et sportifs, etc.

2. Norbert Elias, La civilisation des meeurs, Paris, Calmann-Lévy, 1973 ; La Dynamique de l'Occident, Paris, Calmann-Lévy, 1975.

3. Dans Français et Anglais à table, du Moyen Âge à nos jours, Paris, Flammarion, 1987, S. Mennel cherche à voir comment les changements alimentaires et culinaires relèvent du domaine culinaire mais aussi culturel ou politique et comment ces changements ont influé sur les goûts, l'appétit et la cuisine des Français et des Anglais.

4. Faustin Regnier, L'exotisme culinaire. Essai sur les saveurs de l'Autre, Presses Universitaires de France, Coll. Le Lien Social, Paris, 2004.

5. Eric Hobsbawn, "Inventing traditions", Enquête n² 2, 1995, pp. 171-192.

6. Par "turcité" ou "kurdité", nous entendons l'ensemble des manières dont les immigrés d'origine ethnique turque ou kurde, ainsi que leurs héritiers, imaginent leur appartenance à la communauté turque ou kurde à travers l'alimentation.

7. Cf. "Pratiques alimentaires et identités culturelles", Ethnologie française, $n^{\circ} 1,1997$.

8. Jean-Pierre Corbeau, "Pour une approche plurielle de notre alimentation", Cholédoc, novembre-décembre, $\mathrm{n}^{\circ} 104,2007$.

9. Voir Hatice Soytürk, Florence Strigler et Stéphane De Tapia, "De la biographie des aliments à l'identité culturelle des adolescents", Cahiers de l'Ocha, n¹4, p. 47-56. Cet article plonge dans la diversité de l'univers turc à travers la question des réseaux d'approvisionnement turc en Alsace. Il s'agit d'une étude comparative entre les familles turques et laotiennes vis-à-vis de leur rapport à l'alimentation.

10. Claude Fischler, La formation des goûts alimentaires chez l'enfant et l'adolescent. Rapport de recherche, Paris, DGRST, 1985.

11. Isabelle Garabuau-Moussaoui, Cuisine et indépendance. Jeunesse et alimentation, Paris, L'Harmattan, 2002.

12. Anne Muxel, Individu et mémoire familiale, Paris, Hachette Littératures, 1996.

13. Les adolescents issus de familles religieuses sunnites procèdent à un rituel de remerciement et de purification par la religion, à chaque repas, au début et à la fin. Avant de commencer à consommer, ils disent en arabe "Bismillahirrahmanirrahim", et, à la fin du repas, "Yarrabi ükür, alhamdoulillah".

14. Anthony Giddens, The Consequences of Modernity, Stanford, Stanford University Press, 1990. 